\title{
Emotion Recognition in children with profound and severe deafness: Do they have a deficit in perceptual processing?
}

\author{
Amanda Ludlow ${ }^{1}$, Pam Heaton ${ }^{3}$, Delphine Rosset ${ }^{2}$, Peter Hills ${ }^{1}$ and Christine Deruelle ${ }^{2}$ \\ ${ }^{1}$ Anglia Ruskin University, East Road, Cambridge, CB1 1PT, ${ }^{2}$ INCM, Centre National de Recherche Scientifique, Marseille, \\ France, ${ }^{3}$ Goldsmiths College, University of London, SE14 6NW
}

\begin{abstract}
Findings from several studies have suggested that deaf children have difficulties with emotion identification and that these may impact upon social skills. The authors of these studies have typically attributed such problems to delayed language acquisition and/or opportunity to converse about personal experiences with other people (Peterson \& Siegal, 1995, 1998). The current study aimed to investigate emotion identification in children with varying levels of deafness by specifically testing their ability to recognize perceptual aspects of emotions depicted in upright or inverted human and cartoon faces. The findings from the study showed that, in comparison with both chronological and mental age-matched controls, the deaf children were significantly worse at identifying emotions. However, like controls, their performance decreased when emotions were presented on the inverted faces thus indexing a typical configural processing style. No differences were found across individuals with different levels of deafness or in those with and without signing family members. The results are supportive of poor emotional identification in hearing impaired children and are discussed in relation to delays in language acquisition and inter-group differences in perceptual processing.
\end{abstract}

Keywords: facial expressions, cartoon faces, inversion effect, deafness

It has been suggested that children suffering from deafness manifest difficulties in social skills (e.g., Kusché, Garfield \& Greenberg, 1983). Studies have shown that when children with severe prelingual deafness are not exposed to natural (sign) language from infancy (Isham \& Kamin, 1993), pronounced delays in social knowledge and competence (Kusché et al., 1983; Wiesle \& Bar-Lev, 1992) and social adjustment (Vernon \& Greenberg, 1999) emerge. It has been proposed that these difficulties are a direct effect of delays in language acquisition and missed opportunities to converse with others (e.g., Peterson \& Siegal, $1995 ; 1998)$.

Among the social impairments documented in the deaf population are mentalizing problems and deficits in Theory of Mind (ToM) (i.e., understanding of others' emotions and actions). These difficulties have been observed in congenitally deaf children of hearing non-signing parents (Peterson \& Siegal, 1995, 1999; Steeds, Rowe \& Dowker, 1997; Russell et al., 1998). The importance of language exposure for developing ToM skills is highlighted by findings from studies showing that the ToM deficits observed in deaf children with hearing parents are not in evidence in deaf children who have benefited from the comparative richness of a signing environment (Courtin, 2000; Peterson \& Siegal, 1999).

Another crucial component of social cognition that may be affected by deafness is the ability to understand other people's emotions (e.g., Rieffe \& Terwogt, 2000). Although such deficits are less well documented than mentalizing deficits, findings from several studies suggest that deaf children are more prone to errors in recognizing facial expressions of emotion than their hearing controls. In particular, people with prelingual hearing loss make more errors than people with postlingual hearing loss (Bachara, Raphael \& Phelan, 1980; Schiff, 1973). However, later studies indicated that deaf children can perform as well as hearing children on a simple emotion recognition task when it involved emotion matching rather than emotion recognition (Hosie, Gray, Russell, Scott \& Hunter, 1998; Weisel, 1985). A more recent study, carried out by Dyck, Farrugia, Shochet \& Homes-Brown (2004) compared a group of deaf children and adolescents to an age matched group of vision impaired children and adolescents and ones with no sensory impairment, on a battery of emotion recognition and emotion understanding tasks. This included two tests of ability to recognise vocal expressions of emotion, two tests of the ability to recognise facial expressions of emotion and three tests of emotion understanding. The findings showed that the deficits in emotion recognition, demonstrated in the hearing impaired group, are not stable with age and tend to decrease with age. However, whilst the deaf adolescents demonstrated significantly better performance than deaf children, they still performed at a lower level than age-matched hearing controls. Interestingly, these results revealed emotion recognition deficits in deaf children that were comparable to those previously reported in children with autism. Yet, the mechanisms underlying these emotion recognition deficits in deaf children and adolescents are poorly understood, and it is currently unclear whether they are specific to social stimuli, thereby reflecting their atypical experience, or reflect more general abnormalities in visual perceptual information processing strategies.

A substantial body of research focusing on autistic pathology has shown that an inability to understand facial expressions of emotion is one component of the social deficits characterizing the disorder (e.g., Dakin \& Frith, 2005). Atypical perceptual processing has been proposed as a possible explanation for these emotional impairments. There is evidence to suggest that, whilst typically developing individuals use configural strategies to process faces (e.g., Bukach, Gauthier \& Tarr, 2006) and emotional expressions (e.g., Calder, Keane, Manes, Antoun \& Young, 2000), individuals with Autistic Spectrum Disorders rely on local strategies in both emotion processing (e.g., Rosset et al., 2008) and face processing (Behrmann et al., 2006, Deruelle, Rondan, 
Gepner \& Tardif, 2004). Young children with autism also show a reduced tendency to attend to faces (e.g., Dawson, Webb \& McPartland, 2005) and this has led to the suggestion that atypicalities in face processing reflect poor expertise in face processing.

Interestingly, a similar explanation could hold for deaf children whose experience with faces is also atypical. Face patterns are essential for sign language and many words have the same sign (hand) adverbials whilst differing on facial adverbials (Reilly, McIntire, \& Bellugi, 1990). The facial expressions of signers convey grammatical and lexical distinctions and sign usage may result in an atypical pattern of face processing expertise that is characterised by enhanced featural processing, at the expense of configural processing (Emmorey, 2002). Indeed there is evidence that experience with sign language may enhance certain visual abilities in deaf adults. Bettger, Emmorey, McCullough, and Bellugi (1997) report that native deaf and hearing signers perform significantly better than hearing nonsigners in discriminating human faces photographed under different conditions of orientation and lighting. According to the authors, this result is caused by the greater reliance of signers on the linguistic aspects of facial expression. Therefore, children who reply on lip-reading may differ from hearing children in that their attention may be more directly focused on the mouth region of the face and thus more oriented toward a local face processing style. These conclusions concord with data from McCullough and Emmorey (1997) yielding superior ability to detect subtle differences in facial features in ASL signers than in hearing individuals. However it has also been shown that the level of speech intelligibility influences the area of eye fixation on the face and could also be a contributory factor to how emotions are processed. For example, Buchan, Paré, Munhall, (2002) presented dynamic faces in an emotion recognition task and observed a reduction in eye gaze fixation when there was additional acoustic noise during the test trials. The results showed a lengthening duration of eye gaze on the nose and mouth regions.

The aim of the current study was to determine whether deaf children, who experience difficulties in processing emotions on faces, will manifest atypical perceptual processing strategies (i.e., locally oriented) in response to face stimuli. The experiment includes three different types of faces varying along a human-cartoon continuum. The stimuli were photographs of real faces, human-like cartoon faces and cartoon faces that were not human-like. This continuum provides an easy way to identify the importance of features in processing emotions, such that cartoons clearly contain fewer and more distinguishable features than real faces. Importantly, cartoons may also be processed differently, as social impairments, often found in deaf, are less likely to interfere with this (less-sociable) type of stimuli. As a further test of perceptual strategy, the stimuli were presented in two different orientations (upright and inverted). Any absence of the face inversion effect, whereby faces are better recognised in upright conditions, is taken as a sign of a local rather than a configural processing bias. This is because only local information is available in inverted faces (e.g., Carey \& Diamond, 1994; Deruelle, Rondan, Gepner \& Fagot, 2006).

Importantly a similar study has been carried out with children with ASD, and has shown that processing strategy may differ according to the type of face (Rosset et al., 2008). Whilst both the typically developing controls and ASD groups showed greater overall performance for real faces than the cartoon faces, differences occurred in their processing styles. Whereas the typically developing children relied on a configural processing strategy with all types of faces, the ASD children appear to mirror this strategy only for cartoon faces.

Distinct predictions can be made based on the hypothesis that deaf children may show differences in perceptual processing of emotions. Firstly previous literature has suggested that they have a deficit in the processing of emotions, so one of the aims of this study is to test this finding. If deaf children attend more to local features to process emotions, they may show less of an inversion effect compared to both of the control groups. Whilst it is anticipated that all children will perform better for real faces than for cartoon human and non-human faces, the deaf group may be more even reliant than controls on real faces to process emotions. This could be due to them being more dependent on real faces to bring communicative, linguistic cues.

\section{Method}

\section{Participants}

Twenty-six deaf children, 10 boys and 16 girls, aged between 6 and 16 years and 8 months (mean age $=11$ years 5 months, SD $=3$ years 2 months) participated in the study. The criteria for selection of deaf children was the presence of either severe or profound prelingual hearing loss. Each participant attended one of three mainstream schools with special units for hearing impaired children. Thirteen children were profoundly deaf (hearing loss $>90 \mathrm{db}$ ) and 13 children were severely deaf (hearing loss $>70 \mathrm{db}$ ), meaning that none of the children in the deaf sample could hear conversational speech (approximately 60DB) and consequently did not spontaneously learn to talk. Seven preferred to communicate via sign, thirteen were bilinguals and six preferred to communicate verbally. None of the deaf participants had known associated medical disorders at the time of testing. Mental age scores (Mean mental age $=9$ years 3 months, $\mathrm{SD}=2$ years 4 months) were derived from scores on the Raven Matrices (Raven, Raven \& Court, 1992). A group of 26 control children matched on sex and mental age with the deaf children (MAmatched, aged 6years-13years 4 months: Mean age $=9$ years 3 months, SD $=2$ years 5 months) and a second group of 26 control children matched on sex and chronological age (CA-matched, aged 7years-16years 7 months: Mean age $=11$ years 7 months, $\mathrm{SD}=2$ years 9 months) also participated in the experiment.

\section{Stimuli}

The stimuli were those previously used by Rosset et al. (2008) and by Santos, Rosset \& Deruelle, (2009). They comprised 18 black and white pictures of faces ( 9 female, 9 male) of which 6 were photographs of human faces, 6 were human-like cartoon faces and 6 were cartoon faces that did not look human. Pictures were presented in upright and inverted conditions. Each face was presented in happy, sad and angry conditions, resulting in a total of 54 images (see Figure 1). Human faces were taken from the AR Face Database (Martinez \& Benavente, 1998). Human and non-human cartoon faces were chosen from cartoon movies. All faces were presented in full face presentation and were cropped at the neckline. Pictures subtended approximately $14^{\circ} \mathrm{x} 11^{\circ}$ when viewed at $60 \mathrm{~cm}$. Stimuli were displayed in the centre of a 14 inch computer screen using Microsoft Power Point Presentation software. 


\section{Procedure}

Participants were individually tested in a quiet classroom at their school. They were seated in front of a portable computer screen on which the stimuli were presented and were asked to categorize the emotion displayed. In order to make sure instructions were understood, all participants were presented with 12 training trials. Following this, they were presented with 108 test trials, corresponding to 3 blocks of 36 trials. Each block comprised 18 upright and 18 inverted faces; 6 human faces, 6 faces of human cartoons and 6 faces of non-human cartoons. The order of block presentation was randomized across participants. When necessary, a short pause was proposed between each block. Stimuli were displayed on the screen until the subject responded. Verbal responses to identify the emotion presented on screen were recorded by the experimenter and scored 1 if correct or 0 if incorrect.

Figure 1: Examples of the three different types of faces

Human Face

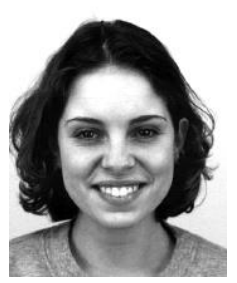

Non-human cartoon

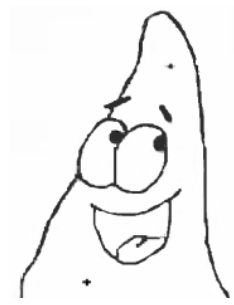

Human Cartoon

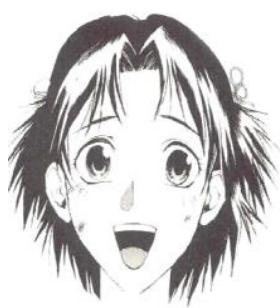

\section{Results}

The number of identification errors was analysed using a 3 × 3 × 2 mixed factorial ANOVA with the factors: subjects (deaf, MA-matched, CA-matched); type of face (real human, cartoon human, and non-human); and orientation of the face (upright and inverted). The between-subjects variable was deaf/MA/CA matched groups, and the within-subjects variables were type and orientation of the face. Table 1 shows the means and standard deviations for the number of errors for type of face and orientation of face across the different participant groups. This analysis revealed a main effect of subjects, $\mathrm{F}(2,75)=23.63, \mathrm{MSE}=18.24$, $\mathrm{p}<$ .001 , partial $\eta^{2}=0.39$. Tukey Post hoc analyses revealed that deaf participants produced significantly more errors than MA (Mean difference $=2.75, \mathrm{p}<.001$ ) or CA participants (Mean difference $=2.99, \mathrm{p}<.001)$ and there was no difference in errors between MA and CA participants (Mean difference $=0.24, \mathrm{p}=.87$ ).

Table 1. Mean number of errors (with standard deviation in parentheses) for participant main effects and the interaction between face type and orientation

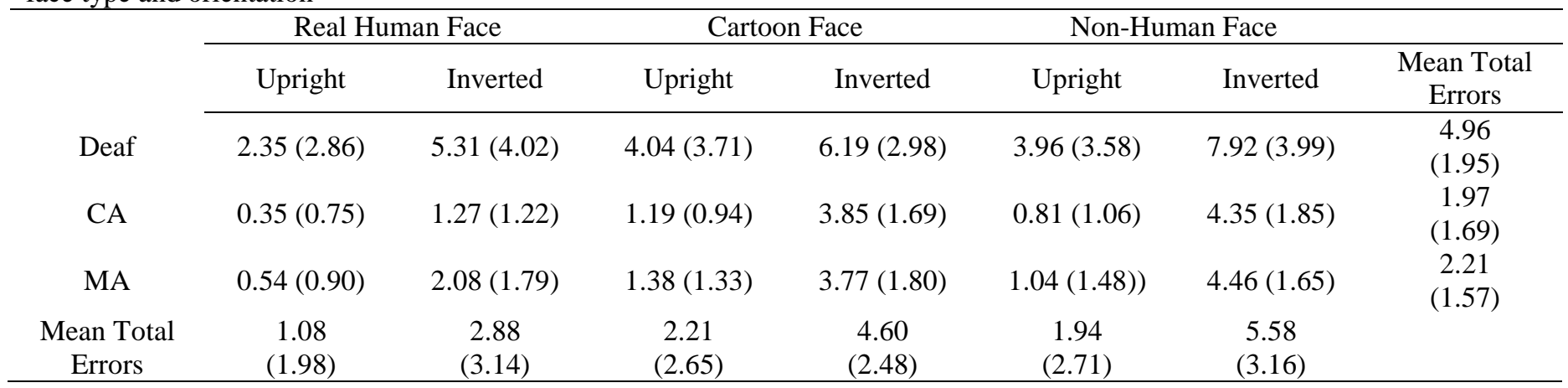

The analysis showed a main effect of type of face, $F(2,150)=41.81$, MSE $=3.02$, $p<.001$, partial $\eta^{2}=0.36$, with fewer errors in response to real human faces than to cartoon human faces (Mean difference $=1.42, \mathrm{p}<.001$ ) and cartoon non-human faces (Mean difference $=1.78, \mathrm{p}<.001$ ). There were no differences in errors to cartoon human faces and cartoon non-human faces $($ Mean difference $=0.35, \mathrm{p}=.12)$. The main effect of orientation was also significant $(\mathrm{F}(1,75)=187.93, \mathrm{MSE}=4.26, \mathrm{p}<$ .001 partial $\eta^{2}=0.72$ ), and fewer errors were made to upright faces than inverted faces (Mean difference $=2.62, p<.001$ ). The interaction between facial orientation and group was not significant $\left(\mathrm{F}(2,75)=1.17, \mathrm{MSE}=4.26, \mathrm{p}=32\right.$ partial $\left.\eta^{2}=0.03\right)$ suggesting that the deaf children did not adopt a local bias when processing the face stimuli.

There was a significant interaction between type of face and orientation, $F(2,150)=16.12, \mathrm{MSE}=2.12, \mathrm{p}<.001$, partial $\eta^{2}=$ 0.18. Analysis using simple effects showed that emotion recognition was poorer for inverted faces than for upright faces in the real human face condition (Mean difference $=1.81, \mathrm{p}<.01$ ). This pattern was repeated for cartoon human faces (Mean difference $=2.40, \mathrm{p}<.005$ ) and for cartoon non-human faces (Mean differences $=3.64, \mathrm{p}<.001$ ).

Critically, there was a significant three-way interaction, $\left(\mathrm{F}(4,150)=2.62, \mathrm{MSE}=2.12, \mathrm{p}<.05\right.$, partial $\eta^{2}=0.07$. $)$ Simple effects demonstrated that deaf participants made fewer errors for upright than inverted real faces, cartoon human faces, and nonhuman faces. This pattern was the same for MA and CA participants, although there was a greater error rate for inverted non- 
human faces than other stimuli (Tukey tests, all ps $<.05$ ). This pattern is reflected in figure 2 . In summary, the results showed that whilst deaf children make more errors overall, their pattern of performance was similar to that of comparison hearing children.

In order to identify the effects of age and IQ on task performance, Spearman Rank correlation tests were computed for each group separately. Results revealed that none of these correlations was significant, (all ps > .05).

Further analyses were carried out on the data from the deaf group. These showed that there was no effect of severity of hearing loss on error rate, $\left(\mathrm{F}(1,24)=0.001, \mathrm{MSE}=263.86, \mathrm{p}=.98\right.$, partial $\left.\eta^{2}=0.01\right)$. As research has shown that deaf children with signing family members perform as well at age and intelligence matched hearing children on emotion recognition and ToM tasks (Courtin, 2000; Peterson \& Siegal, 1999) we compared measured the influence of signing ability of our participants and their signing family members. Total number of errors was compared across the fourteen deaf children who had signing family members (aged 6 years 8 months-15 years 6 months: Mean $=10$ years 4 months, SD $=3.4$ ) with the twelve children with non-signing family members (aged 6 years-16 years 8 months: Mean $=12$ years 9 months, $\mathrm{SD}=2.68$ ). The results showed that the presence of a deaf family member did not appear to influence the results $\left(F(1,24)=1.65\right.$, MSE $=246.94, p=.21$, partial $\left.\eta^{2}=0.06\right)$. Scores for the fifteen children who preferred to communicate using sign sign language (aged 6 years-15 years 7 months: Mean $=10$ years 3 months, SD =3.27). were compared to the eleven deaf children who preferred to communicated orally (aged 8 years-16 years 8 months: Mean $=13$ years 3 months, $\mathrm{SD}=2.46)$, and were found not to differ, $\mathrm{F}(1,24)=0.08, \mathrm{MSE}=262.99, \mathrm{p}=.78, \mathrm{partial} \eta^{2}=$ $0.03)$.

Figure 2: The pattern of errors across the different types of face and their orientation.

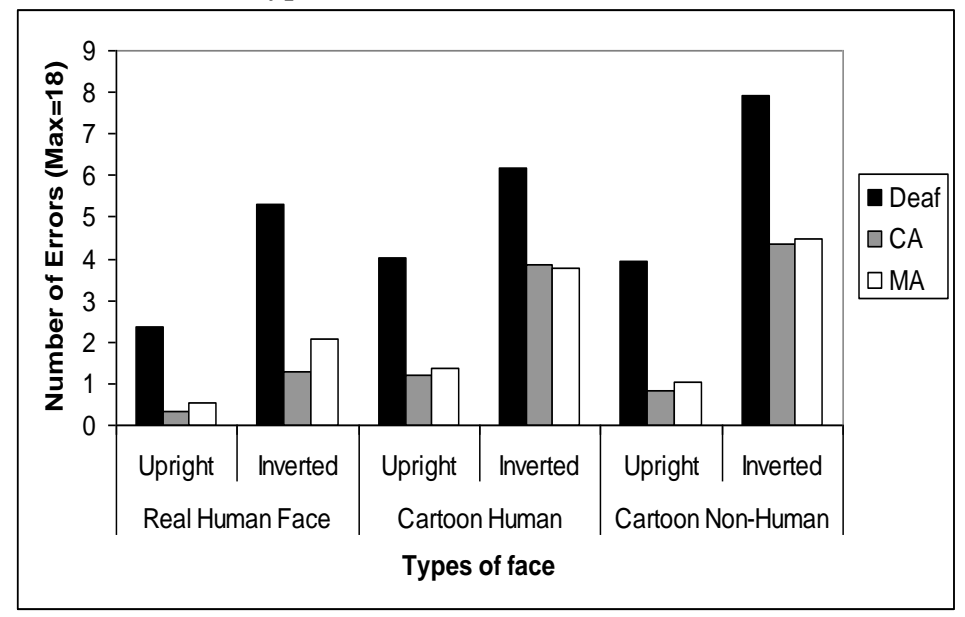

\section{Discussion}

In line with findings from previous research (Bachara et al., 1980; Dyck et al., 2004), the data from the present study showed that deaf children are less able to identify emotions on faces than hearing children matched on chronological and mental age. Whilst deaf children were consistently poorer across the three types of faces, they showed an advantage, similar to hearing controls, for identifying emotions on real faces. One, surprising finding however, given that the ages for the deaf children spanned ten years, is that the age and task performance correlation was not significant. This suggests that results can not be attributed to a delay in ability but rather that they appear to show a relative deficit in emotion recognition. Strikingly however, our findings clearly established that these difficulties do not reflect an atypical, configural visual processing style, characteristic of other developmental disorders such as autism (e.g., Rosset et al., 2008). Successful identification of emotions on inverted faces relies on increased sensitivity to local features and the deaf children, like controls, performed at significantly lower levels on the inverted face conditions. Thus, whilst emotion recognition deficits are in evidence these must be attributed to causal factors other than atypical processing styles.

There is good reason to suppose that when deaf people are looking at faces, they are looking for linguistic information which is less the case for hearing groups (Bettger et al., 1997). However, this may or may not interfere with the configural processing style usually associated with face processing but which is reported to be absent in other groups of children such as those with autism spectrum disorders (Behrmann et al., 2006, Deruelle, Rondan, Gepner \& Tardif, 2004). It is possible that on occasions when deaf people are not communicating, the usual configuration processing of the face takes place and this seems to be supported by the results from the present study. The deaf children, like their hearing controls, were poorer when the faces were inverted. Also, even if experience with sign language is thought to promote featural processing at the expense of configural processing, it should be underlined that many adverbials are stylised and abbreviated facial expressions, whereby the perception of words does in fact involve a configural processing strategy.

An unresolved but important question is whether the deaf children scored at a lower level than the controls in the emotion recognition tasks because only static displays were used. In typical developing individuals the dynamic properties of facial expressions strongly influence their perception (Kamachi et al., 2001). Given that the majority of everyday interactions involve the processing of dynamic faces, it is important to consider how this might preferentially impact upon emotion processing within deaf populations, for whom facial behaviours serve both grammatical and emotion cuing functions. Whilst some studies have suggested that experience with sign language may enhance certain visual abilities (Bettger, et al., 1997) this has largely been 
shown to be specific to linguistic facial expressions. In future studies, therefore, it would be useful to compare emotion recognition in both static and dynamic faces.

Another interesting finding from the current study was that emotion recognition abilities did not distinguish signing and nonsigning participants. A recent study examining the effects of language experience on facial emotion recognition showed that activation patterns within the superior temporal sulcus differed for sign language and spoken language (Emmorey \& McCullough, 2009). This has lead to speculation that both sign language and experience with spoken language may alter the neural organization for recognizing facial expressions. In addition, the strong left-lateralized activation for facial expression recognition previously observed for deaf signers (McCullough, Emmorey, \& Sereno, 2005) was not observed for hearing signers, a finding which also indicates that the condition of deafness itself, and not just signing experience, may impact upon the way that faces are processed in the brain. Therefore the level of signing ability and spoken ability within our deaf sample was of particular relevance to the present results. Yet, our results did not seem to confirm this hypothesis. It should be emphasised however, that our sample sizes were small and this result should be interpreted with caution. In this context, studies carried out with children with cochlear implants may be fundamental in helping us to address questions about emotion processing difficulties in deaf children and the contribution of language to their cognitive development. Indeed, many studies have shown that profoundly deaf children who have received implants before they were 5 years of age are likely to perform better on speech perception and speech production tasks then children receiving implants later on (e.g., Robinson, 1998). If auditory deprivation is responsible for social and emotion deficits through limited ability to communicate with others (e.g., Peterson \& Siegal, 1995, 1998) it would be expected that those fitted with a cochlear implant at an earlier age would show larger gains in both areas. However a recent study of children fitted with a cochlear implant failed to show beneficial effects on visual and auditory emotion recognition tasks (Most \& Aviner, 2009).

Theory of mind deficits have been described in congenitally deaf children (e.g., Peterson \& Siegal, 1995; 1998), and it has been suggested that these reflect delayed language acquisition and fewer opportunities to converse about the experiences of other people. According to Tager-Flusberg \& Sullivan's model of theory of mind (Tager-Flusberg \& Sullivan, 2000) cognitive ToM (understanding of others' emotions and actions) only develops when perceptual ToM (emotion recognition) is intact. This developmental perspective of ToM (perceptual before cognitive) has often been referred to in the literature (Baron-Cohen, 1994; Hobson, 1993). The findings from the current study revealed deficits in emotion recognition in deaf children and these may well contribute to the failure to develop ToM often reported in the deaf population (e.g., Peterson \& Siegal, 1995).

The findings from the present study show that deaf children have emotion recognition deficits despite using a configural processing style. Performance on the task appeared to be related specifically to deafness and level of signing ability did not appear to have a strong influence on the ability to recognize emotions. Whilst the ability to communicate may contribute to poorer emotion identification, future research might consider how limited access to spoken language contributes to the development of visual perceptual strategies for both social and non-social stimuli. This may identify whether poor emotion recognition reflects atypical experience, or reflect abnormalities in visual perceptual information processing strategies.

\section{Acknowledgments}

We would like to thank all the children for taking part in the study and also their parents and teachers for their co-operation in our research. We would also like to express thanks to our anonymous reviewers for their really helpful comments. The work was supported by EU grant (12394) The Stages in the Evolution and Development of Sign Usage.

\section{References}

Bachara, G. H., Raphael, J., \& Phelan, W.J. (1980). Empathy development in deaf preadolescents. American Annals of the Deaf, $125,38-41$.

Baron-Cohen, S. (1994). How to build a baby that can read minds: cognitive mechanisms in mindreading. Current Psychology of Cognition, 13, 513-552.

Bottom of Form

Behrmann, M., Galia Avidan, G. L., Kimchi, R., Luna, B., Humphreys, K., Minshew, N. (2006). Configural processing in autism and its relationship to face processing. Neuropsychologia, 44(1), 110-129.

Bettger, J., Emmorey, K., McCullough, S., \& Bellugi, U. (1997). Enhanced facial discrimination: Effects of experience with American Sign Language. Journal of Deaf Studies and Deaf Education, 2(4), 223-233.

Buchan, J.N., Paré, M., Munhall, K.G. (2007). Spatial statistics of gaze fixations during dynamic face processing. Social Neuroscience,2(1),1-13.

Bukach, C. M., Gauthier, I., \& Tarr, M.J. (2006). Beyond faces and modularity: The power of expertise framework. Trends in Cognitive Neuroscience, 10,159-166.

Calder, A. J., Keane, J., Manes, F., Antoun, N., \& Young, A. W. (2000). Impaired recognition and experience of disgust following brain injury. Neuroscience, 3, 1077-1078.

Carey, S., \& Diamond, R. (1994). Are faces perceived as configurations more by adults than by children? Visual Cognition, 1, 253-274

Courtin, C. (2000). The impact of sign language on the cognitive development of deaf children: The case of theories of mind. Journal of Deaf Studies and Deaf Education, 5, 266-276. 
Dakin, S., \& Frith, U. (2005). Vagaries of Visual Perception in Autism. Neuron, 48(3), 497-507.

Dawson, G., Webb, S. J., \& McPartland, J. (2005). Understanding the nature of face processing impairment in autism: Insights from behavioural and electrophysiological studies. Developmental Neuropsychology, 27, 403-424.

Deruelle, C., Rondan, C., Gepner, B. \& Tardif, C. (2004). Spatial frequency and face processing in children with autism spectrum disorders. Journal of Autism and Developmental Disorders, 34, 199-210.

Deruelle, C., Rondan, C., Gepner, B., Fagot, J., (2006). Processing of compound visual stimuli by children with autism and Asperger syndrome. International Journal of Psychology, 41(2), 97-106.

Dyck, M.J., Farrugia, C., Shochet, I. M., \& Holmes-Brown, M. (2004). Emotion recognition/understanding ability in hearing or vision-impaired children: Do sounds sights, or words make the difference? Journal of Child Psychology and Psychiatry, 45(5), 789-800.

Emmorey, K. (2002). Language, cognition, and the brain: Insights from sign language research. Lawrence Erlbaum and Associates: Mahwah, NJ.

Emmorey, K., \& McCullough, S. (2009). The bimodal bilingual brain: Effects of sign language experience. Brain and Language, 109 (2-3), Pages 124-132

Hosie, J. A., Gray, C.D., Russell, P.A., Scott, C., \& Hunter, N. (1998). The matching of facial expressions by deaf and hearing children and their production and comprehension of emotion labels. Motivation and Emotion, 22(4), 293-313.

Hobson, R. P. (1993). Understanding persons:The role of affect. In S. Baron-Cohen, H. Tager-Flusberg, \& D. J. Cohen, Understanding others minds: perspectives from autism (pp.204-227). Oxford: Oxford University Press.

Isham, W., \& Kamin, L. (1993). Blackness, deafness, IQ, and g. Intelligence, 17, 37-46.

Kamachi, M., Bruce, V, Mukaida, S., Gyoba, J., Yoshikawa, S., Akamatsu, S. (2001). Dynamic properties influence the perception of facial expressions. Perception, 30, 875-887

Kusché, C. A., Garfield, T. S., \& Greenberg, M.T. (1983). The understanding of emotional and social attributions in deaf adolescents. Journal of Clinical Child Psychology, 12, 153-160.

Martinez, M., Benavente, R., (1998). The AR Face Database. CVC Technical Report \#24.

McCullough, S., \& Emmorey, K. (1997). Face processing by deaf ASL signers : Evidence for expertise in distinguished local features. Journal of Deaf Studies and Deaf Education, 2, 212-222.

McCullough, S., Emmorey, K., \& Sereno, M. (2005). Neural organizations for recognition of grammatical and emotional facial expressions in deaf ASL signers and hearing nonsigners. Cognitive Brain Research, 22(2), 193-203.

Most, T., \& Aviner, C. (2009). Auditory, Visual, and Auditory-Visual Perception of Emotions by Individuals with Cochlear Implants, Hearing Aids, and Normal Hearing. The Journal of Deaf Studies and Deaf Education, doi:10.1093/deafed/enp007.

Peterson. C., \& Siegal, M. (1995). Deafness, conversation and theory of mind. Journal of Child Psychology and Psychiatry, 36,459-474.

Peterson, C., \& Siegal, M. (1998). Changing focus on the representational mind. Deaf, autistic and normal hearing children's concepts of false photos, false drawings and false beliefs. British Journal of Developmental Psychology, 16,301-320.

Peterson, C., \& Siegal, M. (1999). Representing inner worlds: Theory of mind in autistic, deaf, and normal hearing children. Psychological Science, 10, 126-129.

Raven, J., Raven, J. C., \& Court, J. H. (1992). The Standard Progressive Matrices. OUP. Oxford Psychologists Press.

Rieffe, C., \& Terwogt, M. (2000). Deaf children's understanding of emotions: Desires take precedence. Journal of Child Psychology and Psychiatry, 41, 601-608.

Reilly, J.S., McIntire, M.L., \& Bellugi, U. (1990). The acquisition of classifier predicates in ASL. In Lucas, C. (ed.). Sign Language research: theoretical issues. Washington, US: Gallaudet University Press. 358-374.

Robinson, K. (1998). Implications of developmental plasticity for the language acquisition of deaf children with cochlear implants. International Journal of Pediatric Otorhinolaryngology, 46 (1-2), 71-80

Rosset, D. B., Rondan, C., Da Fonseca, D., Santos, A., Assouline, B., \& Deruelle, C. (2008). Typical emotion processing for cartoon but not for real faces in children with Autistic Spectrum Disorders. Journal of Autism and Developmental Disorders, 38(5), 919-925.

Russell, P. A., Hosie, J. A., Gray, C. D., Scott, C., Hunter, N., Banks, J.S., \& Macaulay, M. C. (1998). The development of theory of mind in deaf children. Journal of Child Psychology and Psychiatry, 39, 903-910.

Santos, A., Rosset, D., \& Deruelle, C. (2009). Human versus non-human face processing: Evidence from Williams syndrome. Journal of Autism and Developmental Disorders, 39(11), 1552-1559.

Schiff, W. (1973). Social perception in deaf and hearing adolescents. Exceptional Children, 39(4), 289-97, 
Steeds, L., Rowe, K., \& Dowker, A. (1997). Deaf children's understanding of beliefs and desires. Journal of Deaf Studies and Deaf Education, 2, 185-195.

Tager-Flusberg, H., Sullivan, K., (2000). A componential view of theory of mind: Evidence from Williams syndrome. Cognition, $76,59-89$.

Vernon, M., \& Greenberg, S. (1999). Violence in deaf and hard-of-hearing people: A review

of the literature. Aggression and Violent Behaviour, 4, 259-272.

Weisel, A. (1985). Deafness and perception of nonverbal expression of emotion. Perceptual and Motor skills, 61, 515-522.

Weisel, A., \& Bar-Lev, H. (1992). Role taking ability, nonverbal sensitivity, language and social adjustment of deaf adolescents. Educational Psychology, 12(1),3-13. 